\title{
10-Year Trends in Aortic Dissection: Mortality and Weekend Effect within the US Nationwide Emergency Department Sample (NEDS)
}

\author{
James A. Brown, MA, ${ }^{1}$ Bushra Usmani, $M D,{ }^{2}$ George J. Arnaoutakis, $M D,{ }^{3}$ Derek Serna-Gallegos, $\mathrm{MD},{ }^{1,4}$ \\ Konstadinos A. Plestis, MD, ${ }^{5}$ Syed Mahmood Shah, MD, ${ }^{2}$ Forozan Navid, MD, ${ }^{1,4}$ \\ Courtenay Dunn-Lewis, PhD, ${ }^{1}$ Michael J. Singh, MD, ${ }^{4,6}$ Ibrahim Sultan, MD ${ }^{1,4}$ \\ ${ }^{1}$ Division of Cardiac Surgery, Department of Cardiothoracic Surgery, University of Pittsburgh, Pittsburgh, PA, USA; \\ 2Department of Ophthalmology, University of Pittsburgh, Pittsburgh, PA, USA; \\ ${ }^{3}$ Division of Thoracic and Cardiovascular Surgery, University of Florida, Gainesville, FL, USA; \\ ${ }^{4}$ Heart and Vascular Institute, University of Pittsburgh Medical Center, Pittsburgh, PA, USA; \\ JJefferson Aortic Center, Abington - Jefferson Health, Abington, PA, USA; \\ ${ }^{6}$ Division of Vascular Surgery, Department of Surgery, University of Pittsburgh, Pittsburgh, PA, USA
}

\section{ABSTRACT}

Background: This study examined changes in aortic dissection (AD) mortality from 2006 to 2017 and assessed the impact of weekday versus weekend presentation upon mortality.

Methods: This observational study analyzed all records in the Nationwide Emergency Department Sample (NEDS) database. NEDS aggregates discharge data from 984 hospitals in 36 states and the District of Columbia in the United States of America. All patients with thoracic and thoracoabdominal $\mathrm{AD}$ recorded as their principal diagnosis were identified via ICD codes.

Results: Patient characteristics (weekdaylweekend) count: $26,759 \mid 9,640, P=0.016$; age (years): $65.2 \pm 15.8 \mid 64.7$ $\pm 16.2, P=0.016$; women: 11,318 (42.3\%)|4,086 (42.4), $P$ $=0.883$; Charlson comorbidity index: $2.3 \pm 1.7 \mid 2.3 \pm 1.6, P$ $=0.025$. There were $36,399 \mathrm{ED}$ visits with diagnosed $\mathrm{AD}$. Annual AD diagnoses increased by 70\% from 2006 to 2017. From 2012-2017, patients had lower in-hospital mortality (9.9\% versus $11.9 \%, P<0.001$ ) compared with $2006-2011$. Patients reporting during the weekend had higher in-hospital mortality $(11.8 \%$ versus $10.4 \%, P<0.001)$ compared with weekdays. On multivariable analysis, year of presentation remained independently associated with in-hospital mortality, with 2012-2017 being associated with reduced mortality (odds ratio (OR) 0.90, 95\% CI: 0.82, 0.99, $P=0.031$ ), as compared with 2006-2011. Weekend presentation remained independently associated with worse in-hospital mortality (OR $1.17,95 \%$ CI: $1.05,1.29, P=0.003)$ compared with weekday presentation.

Received February 2, 2021;; accepted February 2, 2021.

Correspondence: Ibrabim Sultan, MD, Division of Cardiac Surgery, Department of Cardiothoracic Surgery, University of Pittsburgh, UPMC Center for Thoracic Aortic Disease, Heart and Vascular Institute, University of Pittsburgh Medical Center, 5200 Centre Ave, Suite 715, Pittsburgh, PA, 15232, Telephone 412623-6193, Fax 412-623-3717 (e-mail: sultani@upmc.edu).
Conclusion: Although AD mortality is decreasing, the patients presenting on the weekend were $13 \%$ more likely to die in the hospital compared with patients presenting during the week.

\section{INTRODUCTION}

While uncommon, aortic dissection (AD) is a life-threatening medical emergency that requires prompt intervention - whether pharmacologic, endovascular, or surgical. Following symptom onset, the risk of mortality increases $1-2 \%$ per hour for untreated $\mathrm{AD}$, with roughly $50 \%$ of patients dying within the first 48 hours [Evangelista 2018; Hirst 1958; Pape 2015]. AD also has been associated with significant morbidity, including cardiac tamponade, aortic insufficiency, aortic rupture, aneurysm formation, and various malperfusion syndromes of the central nervous system, abdominal viscera, and/or peripheral extremities [Evangelista 2018]. Thus, it is critical that patients presenting with symptoms of $\mathrm{AD}$ receive timely diagnosis and treatment to maximize their chances of a favorable outcome.

The International Registry of Acute Aortic Dissections (IRAD) reports that in-hospital mortality for Type A AD has significantly decreased from $31 \%$ to $22 \%$ between 1995 and 2013, while in-hospital mortality has remained unchanged (12\%-14\%) for Type B AD over the same time-period [Pape 2015]. However, the IRAD is a consortium of 52 large hospitals in 12 countries, consisting entirely of academic tertiary referral centers; thus, IRAD findings may have limited generalizability to the broader experience of patients with $\mathrm{AD}$ in the United States of America.

The Nationwide Emergency Department Sample (NEDS) targets community emergency departments (ED), spanning all geographic regions of the United States, urban and rural location, teaching and non-teaching status, and all forms of hospital ownership. The NEDS database previously has been utilized to describe a variety of outcomes, including alcoholrelated admissions [White 2018], "out of hospital" cardiac 
arrest [El Asmar 2019], and ocular injury [Ramirez 2018]. As such, the NEDS database may provide useful data for describing outcomes of a representative population of $\mathrm{AD}$ that may be more generalizable to patients across the United States.

The primary aim of this study was to assess the 10-year trends of in-hospital mortality for patients presenting to the ED with $\mathrm{AD}$ over the NEDS database timeframe (20062017), as well as assess the impact of weekday versus weekend presentation upon in-hospital mortality. We hypothesize that due to advances in medical care (and in line with IRAD observations), $\mathrm{AD}$ mortality will decrease; we further hypothesize and that mortality will be higher on weekends than weekdays.

\section{MATERIALS AND METHODS}

Patient population and study design: This was an observational study utilizing the NEDS database, which is part of the group of national databases developed for the Healthcare Cost and Utilization Project. Currently encompassing years from 2006 to 2017, the NEDS database is the largest ED database in the United States, containing greater than 30 million visits annually, aggregating discharge data from 984 hospitals in 36 states and the District of Columbia. (Supplementary Table 1)

All patients who presented to the ED with "dissection of the aorta" were included in the study. These patients were identified by using the International Classification of Diseases, Ninth Revision, Clinical Modification (ICD-9-CM) and the International Classification of Diseases, Tenth Revision, Clinical Modification (ICD-10-CM), depending on the year of the ED visit. Patients with an AD-related code recorded as the principal diagnosis were identified. Codes 441.01 and 441.03 were used to identify patients with ICD-9-CM codes, while codes I71.01 and I71.03 were used to identify patients with ICD-10-CM codes. These codes encompass thoracic and thoracoabdominal aortic dissections; therefore, this study excluded patients with isolated abdominal aortic dissections (441.02 and I71.02) and patients with aortic dissections at unspecified sites (441.00 and I71.00). Henceforth, this study's patient population will be labeled "AD" for convenience.

The primary outcome of interest was in-hospital mortality (either in the ED or inpatient mortality), with year of ED visit (2006-2011 versus 2012-2017) and day of presentation to the ED (weekday versus weekend) being the two primary independent variables of interest. The weekday was defined as Monday through Friday, while the weekend was defined as Saturday or Sunday. This study was approved by the Institutional Review Board of the University of Pittsburgh with waived informed consent. The research team completed relevant Healthcare Cost and Utilization Project training modules and signed the NEDS Data Use Agreement.

Statistical methods and analysis: Differences for baseline demographic and clinical variables were described by year of ED visit (2006-2011 versus 2012-2017) and by day of presentation to the ED (weekday versus weekend). The distributional characteristics for variables were checked for normalcy. Continuous variables were presented as mean \pm standard deviation for normally distributed data, or median and interquartile range (IQR) for non-normally distributed data. Categorical data were summarized using frequency and percentage. All tests were 2-sided with an alpha level of 0.05 considered to indicate statistical significance. All statistical analyses were performed using STATA, version 15.0 (Stata Corporation, College Station, TX).

Student's t-test was used to compare normally distributed continuous variables between groups, and the Wilcoxon rank-sum test was used for non-normally distributed continuous variables. The Chi-squared or Fisher's exact test was used to compare categorical variables between groups, as appropriate. The Cochran-Armitage test for linear trend was used to assess changes in the proportion of in-hospital mortality amongst patients with $\mathrm{AD}$ over the study's timeframe.

Finally, a multivariable logistic regression model was built to identify variables associated with in-hospital mortality for patients presenting with $\mathrm{AD}$. For the logistic regression, year of presentation, day of presentation, age, sex, primary payer, median household income, hospital region, hospital teaching status, and Charlson comorbidity index (CCI) were included in the model. Given the fact that CCI reflects a patient's composite baseline comorbidity, individual comorbidities were not included in the model.

\section{RESULTS}

Baseline demographic and clinical variables: A total of 36,399 ED visits had either thoracic or thoracoabdominal $\mathrm{AD}$ listed as the principal diagnosis, thereby constituting this study's patient population. Figure 1 depicts the total annual $\mathrm{AD}$ from 2006 to 2017; it shows that annual $\mathrm{AD}$ increases over the study's timeframe. (Figure 1) Specifically, the total number of $\mathrm{AD}$ presenting to the $\mathrm{ED}$ per year increased by $70 \%$ over the study's timeframe, from 2,591 in 2006 to 4,409 in 2017.

Dividing the study's timeframe in half, there were 16,600 (45.6\%) patients presenting from 2006 to 2011 and 19,799 (54.4\%) from 2012 to 2017 . Table 1 lists the baseline characteristics for the entire cohort analyzed by years of presentation. (Table 1) During the 2012-2017 timeframe, patients were slightly younger, less likely to be female, more likely to have Medicaid, more likely to be in lowest quartile of median household income, more likely to present to southern and western hospitals, and more likely to present to a metropolitan teaching hospital than in the 2006-2011 timeframe. Conversely, during the 2012-2017 timeframe, there was a higher proportion of comorbidities, higher overall CCI, and patients were more likely to have severe CCI.

The study cohort alternatively was stratified by weekday presentation versus weekend presentation to determine any baseline differences amongst patients by day of presentation. There were $26,759(73.5 \%)$ patients during a weekday (Monday through Friday) and 9,640 (26.5\%) during the weekend (Saturday or Sunday). Table 2 lists baseline characteristics by day of presentation to the ED (weekday versus weekend). (Table 2) During the weekend, patients were slightly younger 
Table 1. Patients with thoracic or thoracoabdominal aortic dissection: emergency department presentations in 2006-2011 compared with 2012-2017

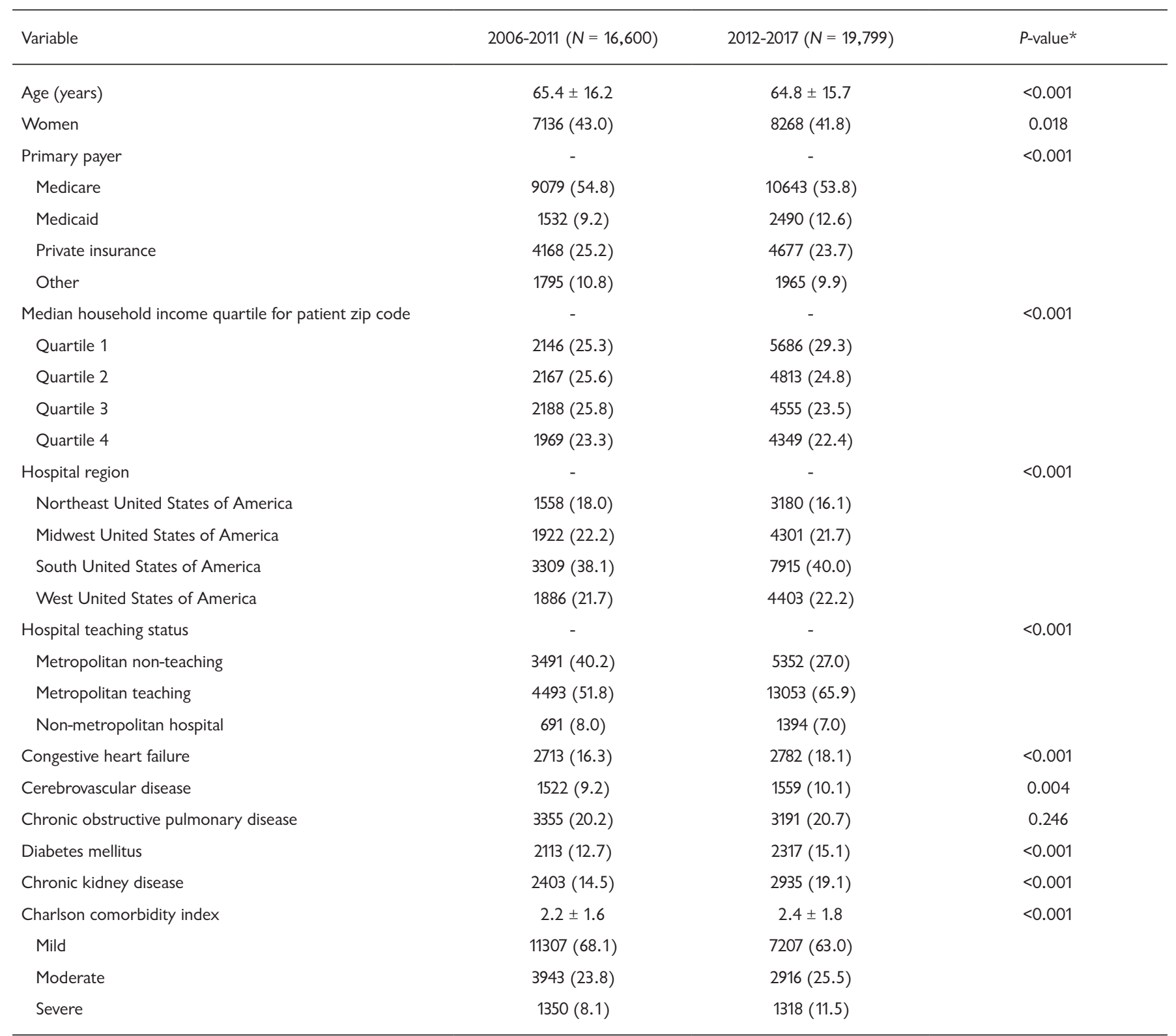

*For continuous variables, $P$-values calculated by the Student's t-test for normally distributed variables or the Wilcoxon rank-sum test for non-normally distributed variables. For categorical variables, $P$-values calculated by the Chi-squared or Fisher's exact test, as appropriate. Values reported as frequency (\%) for categorical variables and mean \pm standard deviation or median (interquartile range) for continuous variables.

and less likely to have chronic kidney disease. However, other demographic variables (including sex, primary payer, median household income, hospital region, and hospital teaching status) as well as other comorbidities (including congestive heart failure, cerebrovascular disease, chronic obstructive pulmonary disease, and diabetes mellitus) were otherwise similar by day of presentation.

In-hospital mortality and total length of stay: For the overall cohort, 3,913 (10.8\%) patients died in the hospital.
Table 3 lists total length of hospital stay and in-hospital mortality, dichotomized by the primary independent variables of interest. From 2012 to 2017, patients with AD had lower overall in-hospital mortality, reduced mortality in the ED, and reduced inpatient mortality compared to 2006 to 2011. (Table 3)

Figure 2 and the Central Illustration depict overall in-hospital mortality for patients with $\mathrm{AD}$ by year of presentation. (Figure 2) Specifically, the proportion of in-hospital mortality 
Table 2. Patients with thoracic or thoracoabdominal aortic dissection: emergency department presentations on weekdays compared with weekends.

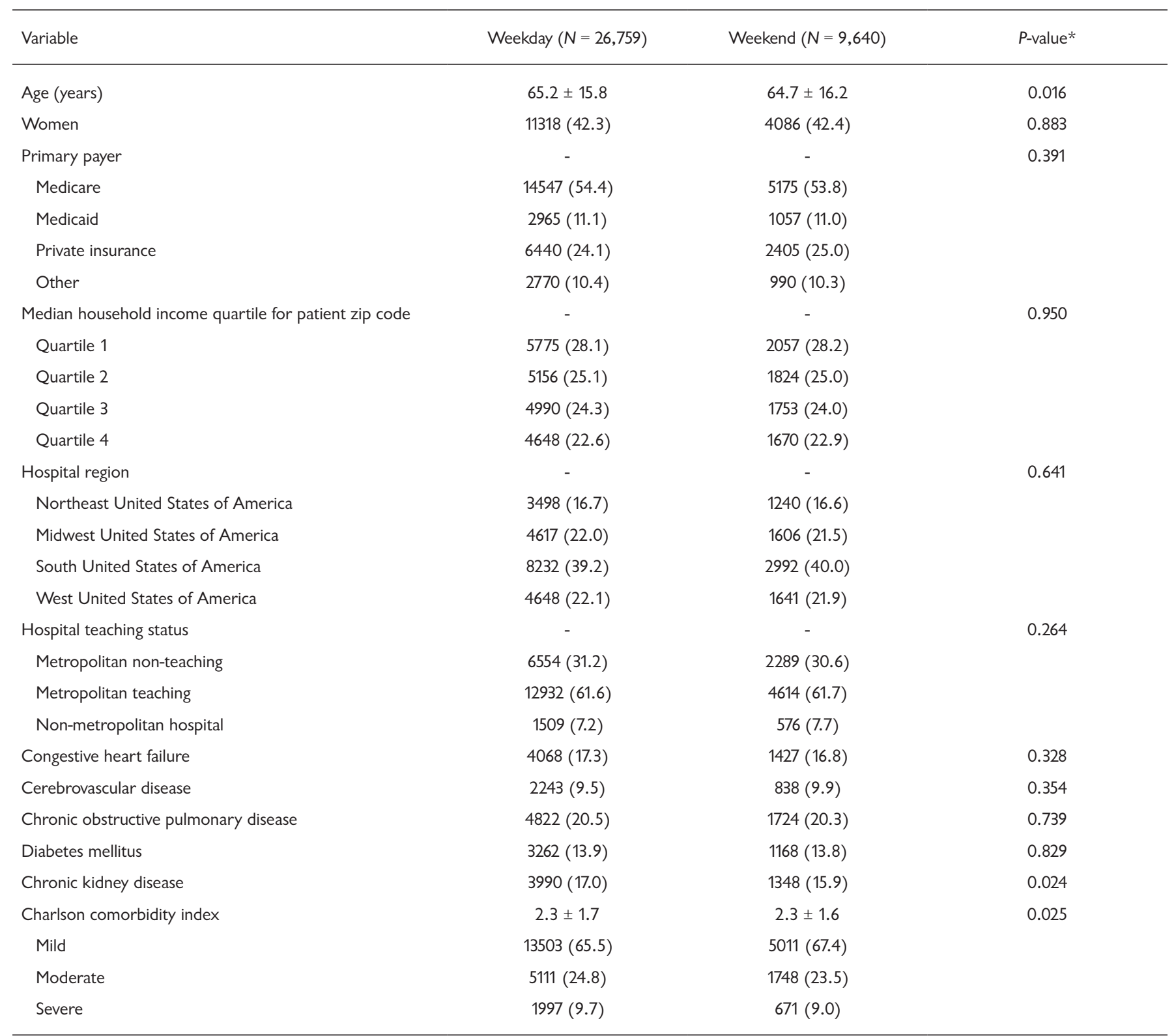

*For continuous variables, $P$-values calculated by the Student's t-test for normally distributed variables or the Wilcoxon rank-sum test for non-normally distributed variables. For categorical variables, p-values calculated by the Chi-squared or Fisher's exact test, as appropriate. Values reported as frequency (\%) for categorical variables and mean \pm standard deviation or median (interquartile range) for continuous variables.

decreased by $30 \%$ over the study's timeframe, from $13.3 \%$ in 2006 to $9.3 \%$ in 2017 , with a significant linear trend over time $(P<0.001$, Cochran-Armitage test). Yet, there was similar total length of hospital stay $(8.3 \pm 11.0$ versus $8.2 \pm 10.6$, $P=0.355)$ for each timeframe (2012-2017 versus 2006-2011).

During the weekend (Table 3), patients with AD had higher overall in-hospital mortality $(11.8 \%$ versus $10.4 \%)$ and worse inpatient mortality (10.1\% versus $9.0 \%)$ even though there was similar mortality in the $\operatorname{ED}(P=0.068)$. Figure 3 depicts overall in-hospital mortality for patients with $\mathrm{AD}$ by day of presentation. (Figure 3) For patients presenting during the weekend, total length of hospital stay was slightly longer compared with patients presenting on a weekday.

Table 4 presents the demographic and clinical variables associated with in-hospital mortality for the overall cohort. (Table 4) On multivariable analysis, year of presentation remained independently associated with in-hospital mortality, with 2012-2017 being associated with reduced mortality 


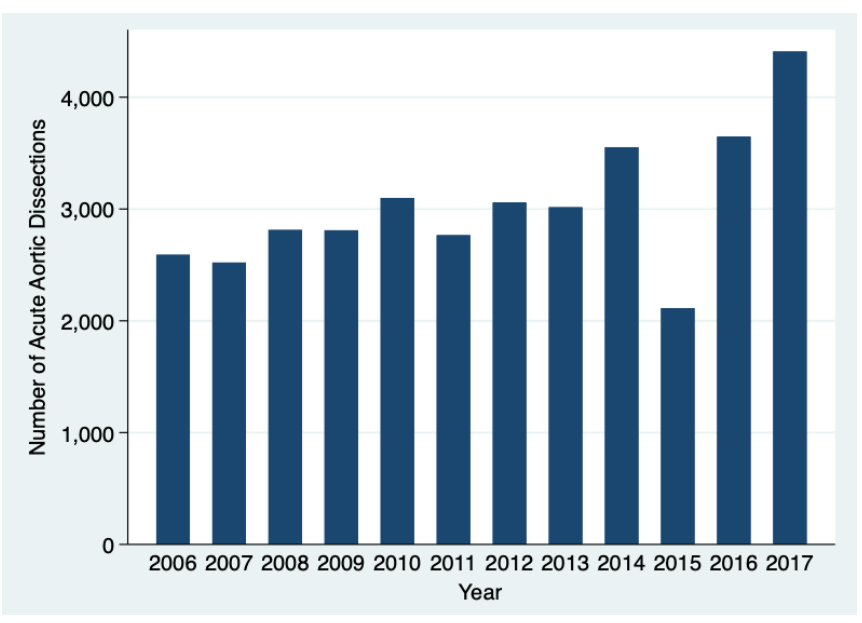

Figure 1. Total number of patients presenting to the emergency department with thoracic or thoracoabdominal aortic dissection by year of presentation.

(odds ratio (OR) 0.90, 95\% CI: $0.82,0.99, P=0.031$ ), as compared with 2006-2011. Moreover, weekend presentation remained independently associated with worse in-hospital mortality (OR 1.17, 95\% CI: 1.05, 1.29, $P=0.003$ ) as compared with weekday presentation. Other significant predictors of in-hospital mortality included age, primary payer, hospital region, hospital teaching status, and Charlson comorbidity index.

\section{DIsCUSSION}

While $\mathrm{AD}$ has a reported incidence as low as 4.4 cases per 100,000 person-years [DeMartino 2018], AD is nonetheless a life-threatening medical emergency that requires timely recognition, diagnosis, and treatment to maximize the chances of a favorable outcome. Utilizing data derived from the NEDS database, this study sought to determine if in-hospital mortality has changed over the timeframe encompassed by the NEDS database (2006-2017). Several important findings emerge from this data that might inform cardiovascular specialists, emergency room physicians, and systems-level quality improvement initiatives.

First, the total number of $\mathrm{AD}$ presenting to the ED per year increased by $70 \%$ over the study's timeframe. Second, in-hospital mortality decreased $16.8 \%$ over the study's timeframe (10\% decreased odds of mortality after 2012), despite a higher proportion of comorbidities in more recent years. Third, there may be a "weekend effect" given the $13 \%$ increase (17\% increased odds) of in-hospital mortality during the weekend (as compared with weekdays), despite the similar proportion of baseline comorbidities across day of presentation. Finally, both principal findings (i.e., improved mortality over the study's timeframe and the "weekend effect") remained even after multivariable risk-adjustment.

While the trend over time ought to encourage physicians caring for patients with $\mathrm{AD}$, the "weekend effect" highlights

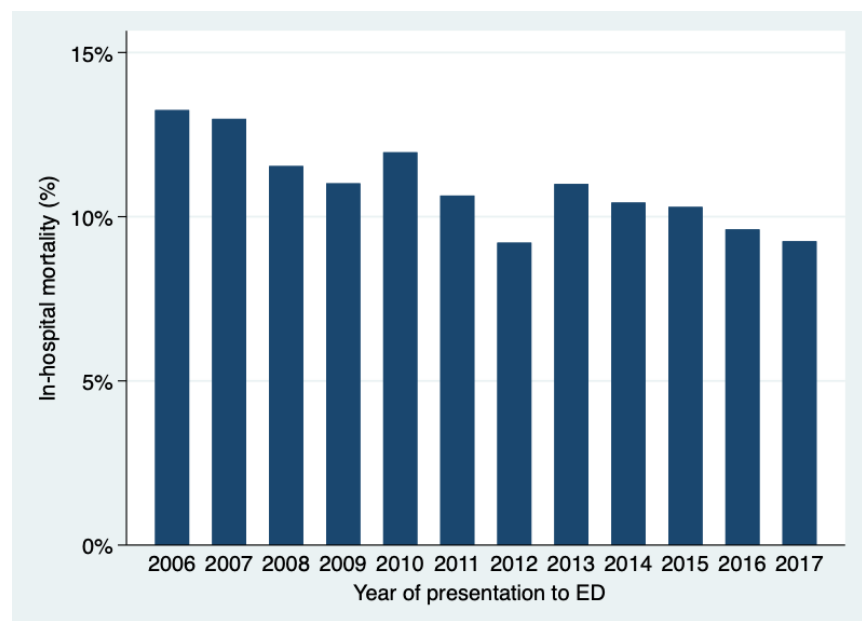

Figure 2. In-hospital mortality for patients presenting to the emergency department with thoracic or thoracoabdominal aortic dissection by year of presentation.

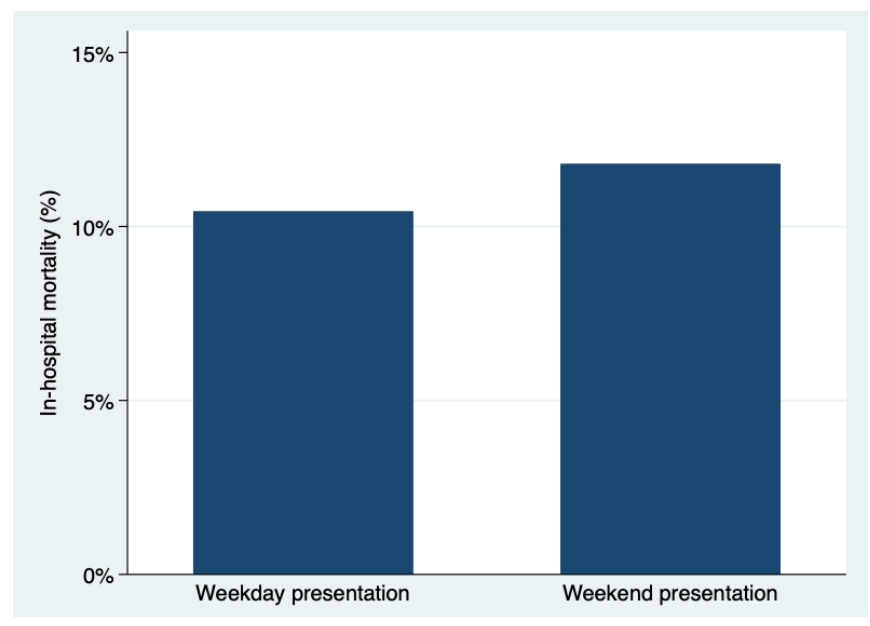

Figure 3. In-hospital mortality for patients presenting to the emergency department with thoracic or thoracoabdominal aortic dissection by weekday versus weekend.

an area for improving outcomes of this highly morbid and potentially lethal disease.

For the timeframe contemporaneous with this study (2006 onward), the IRAD database reports that in-hospital mortality for Type A AD ranges from $20.8 \%$ to $21.7 \%$, while inhospital mortality ranges from $7.7 \%$ to $14.1 \%$ for Type B AD [Pape 2015]. Conversely, other studies suggest that mortality may even be as low as 9\% for AD [David 1999; Mehta 2002; Moon 2001; Zierer 2007; Lai 2002; Chiappini 2005; Lawton 2015; Abe 2020]. In this study of NEDS, $10.8 \%$ of patients with $\mathrm{AD}$ died in the hospital, which resides in the lower end of the range reported in the literature.

There are numerous potential reasons for this discrepancy. First, this study was unable to distinguish Type A from Type $\mathrm{B} \mathrm{AD}$, with the latter type of dissection having lower mortality than the former. ICD codes distinguish thoracic aortic 
Table 3. Patients with thoracic or thoracoabdominal aortic dissection: Total length of hospital stay (LOS) and in-hospital mortality for patients presenting to the emergency department by year (3A) and day of the week (3B).

\begin{tabular}{|c|c|c|c|}
\hline \multicolumn{4}{|l|}{$3 \mathrm{~A}$} \\
\hline Variable & $2006-2011(N=16,600)$ & $2012-2017(N=19,799)$ & $P$-value* \\
\hline Total length of stay (days) & $8.2 \pm 10.6$ & $8.3 \pm 11.0$ & 0.355 \\
\hline In-hospital mortality & $1961(11.9)$ & $1952(9.9)$ & $<0.001$ \\
\hline Died in the ED & $273(1.7)$ & $272(1.4)$ & 0.034 \\
\hline Died in the hospital & $1688(10.2)$ & $1680(8.5)$ & $<0.001$ \\
\hline \multicolumn{4}{|l|}{ 3B } \\
\hline Variable & Weekday $(N=26,759)$ & Weekend $(N=9,640)$ & $P$-value* \\
\hline Total length of stay (days) & $8.1 \pm 10.6$ & $8.5 \pm 11.2$ & 0.013 \\
\hline In-hospital mortality & $2781(10.4)$ & $1132(11.8)$ & $<0.001$ \\
\hline Died in the ED & $382(1.4)$ & $163(1.7)$ & 0.068 \\
\hline Died in the hospital & $2399(9.0)$ & $969(10.1)$ & 0.002 \\
\hline
\end{tabular}

*For continuous variables, p-values calculated by the Student's t-test for normally distributed variables or the Wilcoxon rank-sum test for non-normally distributed variables. For categorical variables, $P$-values calculated by the Chi-squared or Fisher's exact test, as appropriate. Values reported as frequency (\%) for categorical variables and mean \pm standard deviation or median (interquartile range) for continuous variables. ED, emergency department; LOS, length of stay

Table 4. Multivariable logistic regression model for in-hospital mortality of patients presenting with thoracic or thoracoabdominal aortic dissection

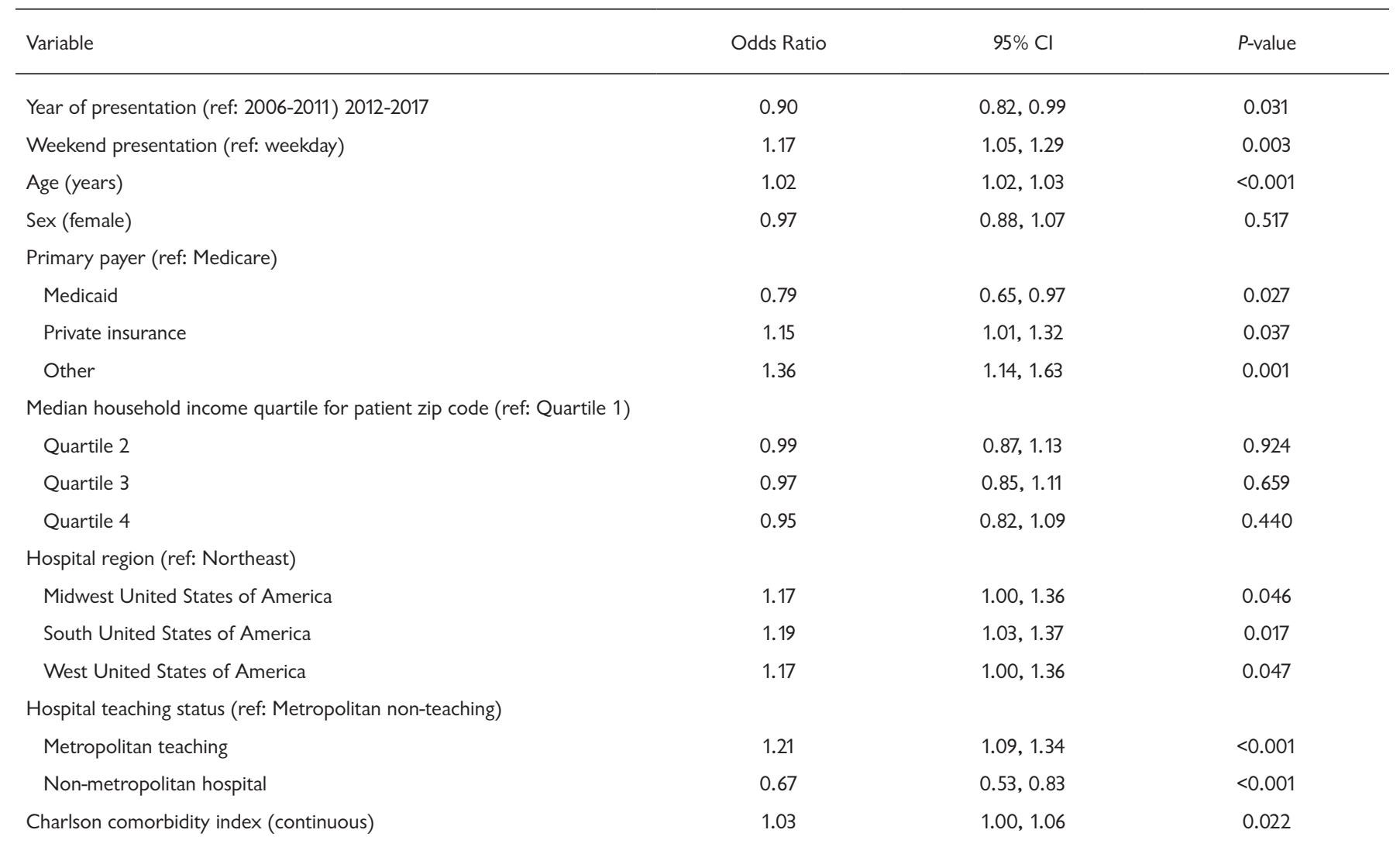


dissections (encompassing DeBakey Type II and Type III A) from thoracoabdominal aortic dissections (encompassing DeBakey Type I and Type III B), as opposed to distinguishing Type A and Type B AD. Moreover, the ICD codes were similarly unable to distinguish acute from chronic $\mathrm{AD}$; however, prior reports suggest that chronic $\mathrm{AD}$ only represents $2.6 \%$ of all AD [DeMartino 2018]. By implication, the overall mortality rate may be lower than expected because this study's population includes Type B with Type A and includes acute with chronic $\mathrm{AD}$.

Second, the NEDS database does not include outcomes of patients who were transferred from one hospital to another, which may have higher mortality. Furthermore, it is possible that the patients recorded in the NEDS are less complex and less acutely ill than the patients recorded in the IRAD database (large tertiary referral centers), potentially contributing to the lower mortality rate reported in NEDS. Alternatively, the population represented in the NEDS database may harbor some selection bias - with patients who die prior to the ED not being recorded in NEDS and patients with favorable outcomes who survive to the ED being included in the database. Whatever the explanation, it is nevertheless possible that this study's mortality rate of $10.8 \%$ may more accurately reflect outcomes of AD for all-comers in the general U.S. population.

According to IRAD [Evangelista 2018; Pape 2015], the National Inpatient Sample [Chikwe 2013; Zimmerman 2016], and other single-institution studies [David 1999; Lawton 2015], short-term mortality of AD has improved over time, especially for Type A. This study of NEDS has similarly identified a trend in improved mortality for patients presenting to the $\mathrm{ED}$ with $\mathrm{AD}$. After multivariable riskadjustment, patients presenting with $\mathrm{AD}$ during 2012-2017 were $10 \%$ decreased odds of dying in the hospital compared to 2006-2011. Notably, this trend appears to occur even though patients presented with higher proportion of comorbidities in more recent years (2012-2017). Prior studies have argued that the reductions in short-term mortality of $\mathrm{AD}$ over time may be due to more sensitive diagnostic modalities, improved systems-level deployment of rapid intervention, and enhanced multidisciplinary care [Evangelista 2018]. Perhaps most importantly, advances in operative technique and approach have reduced surgical mortality for Type A and Type B AD over time [David 1999; Mehta 2002; Moon 2001; Zierer 2007; Lai 2002; Chiappini 2005; Lawton 2015; Abe 2020; Dufendach 2019; Sultan 2018; Sultan 2016; Trivedi 2016; Vallabhajosyula 2017; Sultan 2017; Sultan 2016; Brown 2020; Brown 2020], with endovascular interventions for Type $\mathrm{B}$ providing even greater gains in operative mortality [Fattori 2008; Tolenaar 2014; Trimarchi 2006]. Regardless of cause, the reduction in mortality over time is an encouraging trend for the management of $\mathrm{AD}$.

In addition to the advances noted above, numerous other modifiable risk factors have been studied to improve outcomes of AD. Aortic surgery performed "out of hours" (i.e., during the weekend or at night) has garnered recent attention, with some studies finding an adverse impact on mortality [Ahlsson 2019; Groves 2014; Qiu 2018], while other studies have
Supplementary Table 1. States participating in the 2017 Nationwide Emergency Department Sample (NEDS) database. The percentage of the U.S. population accounted for by NEDS is $80.9 \%$, from $74.8 \%$ in the northeast to $85.4 \%$ in the Midwest.

\begin{tabular}{|c|c|}
\hline State & HCUP Data Organization \\
\hline AR & Arkansas Department of Health \\
\hline$A Z$ & Arizona Department of Health Services \\
\hline CA & Office of Statewide Health Planning and Development \\
\hline $\mathrm{CO}$ & Colorado Hospital Association \\
\hline CT & Connecticut Hospital Association \\
\hline DC & District of Columbia Hospital Association \\
\hline $\mathrm{FL}$ & Florida Agency for Health Care Administration \\
\hline GA & Georgia Hospital Association \\
\hline IA & lowa Hospital Association \\
\hline IL & Illinois Department of Public Health \\
\hline IN & Indiana Hospital Association \\
\hline KS & Kansas Hospital Association \\
\hline KY & Kentucky Cabinet for Health and Family Services \\
\hline MA & Massachusetts Center of Health Information and Analysis \\
\hline MD & Maryland Health Services Cost Review Commission \\
\hline ME & Maine Health Data Organization \\
\hline $\mathrm{MN}$ & Minnesota Hospital Association \\
\hline MO & Missouri Hospital Industry Data Institute \\
\hline MS & Mississippi State Department of Health \\
\hline MT & Montana Hospital Association \\
\hline NC & North Carolina Department of Health and Human Services \\
\hline ND & North Dakota (data provided by the Minnesota Hospital Association) \\
\hline NE & Nebraska Hospital Association \\
\hline NJ & New Jersey Department of Health \\
\hline NV & Nevada Department of Health and Human Services \\
\hline NY & New York State Department of Health \\
\hline $\mathrm{OH}$ & Ohio Hospital Association \\
\hline OR & $\begin{array}{c}\text { Oregon Association of Hospitals and Health Systems, Oregon } \\
\text { Office of Health Analytics }\end{array}$ \\
\hline $\mathrm{RI}$ & Rhode Island Department of Health \\
\hline SC & South Carolina Revenue and Fiscal Affairs Office \\
\hline SD & South Dakota Association of Healthcare Organizations \\
\hline $\mathrm{TN}$ & Tennessee Hospital Association \\
\hline UT & Utah Department of Health \\
\hline VT & Vermont Association of Hospitals and Health Systems \\
\hline WI & Wisconsin Department of Health Services \\
\hline WY & Wyoming Hospital Association \\
\hline
\end{tabular}

Adapted from: 2017 Introduction to the NEDS. Healthcare Cost and Utilization Project (HCUP). August 2020. Agency for Healthcare Research and Quality, Rockville, MD. www.hcup-us.ahrq.gov/db/nation/neds/NEDS_Introduction_2017.jsp 
found no difference [Mehta 2005; Arnaoutakis 2020]. In this study, patients presenting on the weekend had $17 \%$ increased odds of dying compared with patients presenting during the week after multivariable risk-adjustment.

Numerous explanations might explain this "weekend effect" on mortality. First, it is possible that patients may present with a different proportion of comorbidities or demographic characteristics; however, in this study, baseline demographic and clinical variables were similar across the weekend group and the weekday group. Conversely, it is possible that weekend providers may be more fatigued, hospital staffing may be limited, or there may be diagnostic and management delays. Moreover, clinical decision-making may be different during the weekend - i.e., there may be different rates of hemiarch, total arch, or frozen elephant trunk operations for Type A AD or there may be different rates of medical versus endovascular versus surgical management of Type B AD.

This study suggests another potential explanation for the weekend effect when examining ED versus hospital mortality. When focusing only on ED mortality, the weekend was not statistically different than weekdays. Meanwhile, inpatient hospital mortality was significantly worse $(10.1 \%$ versus $9.0 \%$ ) during the weekend. This may suggest that patients who report on weekdays are receiving more timely diagnosis or rapid consultation with the appropriate inpatient services than weekend patients. Whatever the explanation for the "weekend effect," this study highlights an opportunity for systems-level quality improvement, including dedicated multidisciplinary aortic surgery teams, 24-7 "on-call" staffing and resources (e.g. neurophysiology and perfusion teams), and institutional protocols for treating $\mathrm{AD}$ [Andersen 2016].

\section{LIMITATIONS}

There are important limitations to this analysis. First, this study utilized existing observational data; as such, this cohort is heterogeneous in terms of baseline patient characteristics, standards of care, and clinical decision-making, as well as operative technique and approach across participating institutions. Second, this study lacks certain granular data that might help unearth explanations for the overall mortality findings, including time from symptom onset to treatment, treatment specific variables (medical versus surgical versus endovascular), and operative differences (e.g., extent of proximal and distal repair as well as central nervous system protection strategies). Third, this study was unable to determine the outcomes of patients that were transferred from one hospital to another, which is a clinically relevant subgroup of patients with $\mathrm{AD}$.

Fourth, this study was unable to distinguish Type A from Type $\mathrm{B} A D$, which is a meaningful distinction that entails differing natural history, dictates differing treatment paradigms, and consequently has implications for outcomes. Specifically, CPT codes that define specific treatments would not only serve as surrogate data for Stanford classification, but also would help elucidate trends in clinical decision-making and outcomes after each treatment modality; however, CPT data was unavailable. Moreover, the ICD codes were similarly unable to distinguish acute from chronic $\mathrm{AD}$; however, prior reports suggest that chronic $\mathrm{AD}$ only represents $2.6 \%$ of all AD [DeMartino 2018].

Finally, it should be noted that "big data" analyses with extremely large sample sizes, such as this study of the NEDS database, tend to detect even small magnitudes of differences in the data. Statistical significance may not have clinical relevance, however. For example, weekend mortality rose by $13 \%$ in this study (from $10.4 \%$ to $11.8 \%$ ) - a statistically significant finding that also contradicts prior reports [Mehta 2005; Arnaoutakis 2020]. Further research could determine whether this is clinically meaningful or if this finding merely is a statistical artifact of an over-powered dataset. Nevertheless, while this study should be augmented by findings from single-institutional analyses with the availability of more granular data, this study provides relevant information concerning national mortality data in a diverse, but representative, sample population that may be generalizable to patients with $\mathrm{AD}$.

To our knowledge, this is the first study utilizing the NEDS database to report nationwide outcomes and trends in $\mathrm{AD}$. This may be relevant to cardiovascular specialists, emergency medicine doctors, and systems-level administrators in the United States of America. The total number of AD presenting to the ED per year is significantly increasing over time. Moreover, there may be a "weekend effect" for AD with patients having worse in-hospital mortality during the weekend compared to weekdays. Nevertheless, the proportion of patients with in-hospital mortality is significantly decreasing over time. While this improvement ought to encourage physicians, these findings highlight the weekend as an important focus for improving outcomes of this highly morbid and potentially lethal disease.

\section{REFERENCES}

Abe T, Yamamoto H, Miyata H, et al. 2020. Patient trends and outcomes of surgery for type A acute aortic dissection in Japan: an analysis of more than 10000 patients from the Japan Cardiovascular Surgery Database. Eur J Cardiothorac Surg. 57(4):660-667.

Ahlsson A, Wickbom A, Geirsson A, et al. 2019. Is There a Weekend Effect in Surgery for Type A Dissection?: Results From the Nordic Consortium for Acute Type A Aortic Dissection Database. Ann Thorac Surg. 108(3):770-776.

Andersen ND, Benrashid E, Ross AK, et al. 2016. The utility of the aortic dissection team: outcomes and insights after a decade of experience. Ann Cardiothorac Surg. 5(3):194-201.

Arnaoutakis G, Bianco V, Estrera AL, et al. 2020. Time of day does not influence outcomes in acute type A aortic dissection: Results from the IRAD. J Card Surg.

Brown JA, Arnaoutakis GJ, Kilic A, Gleason TG, Aranda-Michel E, Sultan I. 2020. Current trends in the management of acute type A aortic intramural hematoma. J Card Surg. 35(9):2331-2337.

Brown JA, Arnaoutakis GJ, Kilic A, Gleason TG, Aranda-Michel E, Sultan I. 2020. Medical and surgical management of acute type B aortic intramural hematoma. J Card Surg. 35(9):2324-2330. 
Chiappini B, Schepens M, Tan E, et al. 2005. Early and late outcomes of acute type A aortic dissection: analysis of risk factors in 487 consecutive patients. Eur Heart J. 26(2):180-186.

Chikwe J, Cavallaro P, Itagaki S, Seigerman M, Diluozzo G, Adams DH. 2013. National outcomes in acute aortic dissection: influence of surgeon and institutional volume on operative mortality. Ann Thorac Surg. 95(5):1563-1569

David TE, Armstrong S, Ivanov J, Barnard S. 1999. Surgery for acute type A aortic dissection. Ann Thorac Surg. 67(6):1999-2001; discussion 2014-1999.

DeMartino RR, Sen I, Huang Y, et al. 2018. Population-Based Assessment of the Incidence of Aortic Dissection, Intramural Hematoma, and Penetrating Ulcer, and Its Associated Mortality From 1995 to 2015. Circ Cardiovasc Qual Outcomes. 11(8):e004689.

Dufendach KA, Sultan I, Gleason TG. 2019. Distal Extent of Surgery for Acute Type A Aortic Dissection. Operative Techniques in Thoracic and Cardiovascular Surgery. 24(2):82-102.

El Asmar A, Dakessian A, Bachir R, El Sayed M. 2019. Out of hospital cardiac arrest outcomes: Impact of weekdays vs weekends admission on survival to hospital discharge. Resuscitation. 143:29-34.

Evangelista A, Isselbacher EM, Bossone E, et al. 2018. Insights From the International Registry of Acute Aortic Dissection: A 20-Year Experience of Collaborative Clinical Research. Circulation. 137(17):1846-1860.

Fattori R, Tsai TT, Myrmel T, et al. 2008. Complicated acute type B dissection: is surgery still the best option?: a report from the International Registry of Acute Aortic Dissection. JACC Cardiovasc Interv. 1(4):395-402.

Groves EM, Khoshchehreh M, Le C, Malik S. 2014. Effects of weekend admission on the outcomes and management of ruptured aortic aneurysms. J Vasc Surg. 60(2):318-324.

Hirst AE, Jr., Johns VJ, Jr., Kime SW, Jr. 1958. Dissecting aneurysm of the aorta: a review of 505 cases. Medicine (Baltimore). 37(3):217-279.

Lai DT, Robbins RC, Mitchell RS, et al. 2002. Does profound hypothermic circulatory arrest improve survival in patients with acute type a aortic dissection? Circulation. 106 (12 Suppl 1):I218-228.

Lawton JS, Liu J, Kulshrestha K, et al. 2015. The impact of surgical strategy on survival after repair of type A aortic dissection. J Thorac Cardiovasc Surg. 150(2):294-301 e291.

Mehta RH, Manfredini R, Bossone E, et al. 2005. Does circadian and seasonal variation in occurrence of acute aortic dissection influence inhospital outcomes? Chronobiol Int. 22(2):343-351.

Mehta RH, Suzuki T, Hagan PG, et al. 2002. Predicting death in patients with acute type a aortic dissection. Circulation. 105(2):200-206.

Moon MR, Sundt TM, 3rd, Pasque MK, et al. 2001. Does the extent of proximal or distal resection influence outcome for type A dissections? Ann Thorac Surg. 71(4):1244-1249; discussion 1249-1250.
Pape LA, Awais M, Woznicki EM, et al. 2015. Presentation, Diagnosis, and Outcomes of Acute Aortic Dissection: 17-Year Trends From the International Registry of Acute Aortic Dissection. J Am Coll Cardiol. 66(4):350-358.

Qiu J, Zhang L, Luo X, et al. 2018. Higher Mortality in Patients Undergoing Nighttime Surgical Procedures for Acute Type A Aortic Dissection. Ann Thorac Surg. 106(4):1164-1170.

Ramirez DA, Porco TC, Lietman TM, Keenan JD. 2018. Ocular Injury in United States Emergency Departments: Seasonality and Annual Trends Estimated from a Nationally Representative Dataset. Am J Ophthalmol. 191:149-155.

Sultan I, Bavaria JE, Szeto W. 2016. Hybrid Techniques for Aortic Arch Aneurysm Repair. Semin Cardiothorac Vasc Anesth. 20(4):327-332.

Sultan I, Bianco V, Yazji I, et al. 2018. Hemiarch Reconstruction Versus Clamped Aortic Anastomosis for Concomitant Ascending Aortic Aneurysm. Ann Thorac Surg. 106(3):750-756.

Sultan I, McGarvey J, Vallabhajosyula P, Desai ND, Bavaria JE, Szeto WY. 2016. Routine use of hemiarch during acute type A aortic dissection repair. Ann Cardiothorac Surg. 5(3):245-247.

Sultan I, Wallen TJ, Habertheuer A, et al. 2017. Concomitant antegrade stent grafting of the descending thoracic aorta during transverse hemiarch reconstruction for acute DeBakey I aortic dissection repair improves aortic remodeling. J Card Surg. 32(9):581-592.

Tolenaar JL, Froehlich W, Jonker FH, et al. 2014. Predicting in-hospital mortality in acute type B aortic dissection: evidence from International Registry of Acute Aortic Dissection. Circulation. 130(11 Suppl 1):S45-50.

Trimarchi S, Nienaber CA, Rampoldi V, et al. 2006. Role and results of surgery in acute type B aortic dissection: insights from the International Registry of Acute Aortic Dissection (IRAD). Circulation. 114(1 Suppl):I357-364.

Trivedi D, Navid F, Balzer JR, et al. 2016. Aggressive Aortic Arch and Carotid Replacement Strategy for Type A Aortic Dissection Improves Neurologic Outcomes. Ann Thorac Surg. 101(3):896-903; Discussion 903-895.

Vallabhajosyula P, Gottret JP, Menon R, et al. 2017. Central Repair With Antegrade TEVAR for Malperfusion Syndromes in Acute Debakey I Aortic Dissection. Ann Thorac Surg. 103(3):748-755.

White AM, Slater ME, Ng G, Hingson R, Breslow R. 2018. Trends in Alcohol-Related Emergency Department Visits in the United States: Results from the Nationwide Emergency Department Sample, 2006 to 2014. Alcohol Clin Exp Res. 42(2):352-359.

Zierer A, Moon MR, Melby SJ, et al. 2007. Impact of perfusion strategy on neurologic recovery in acute type A aortic dissection. Ann Thorac Surg. 83(6):2122-2128; discussion 2128-2129.

Zimmerman KP, Oderich G, Pochettino A, et al. 2016. Improving mortality trends for hospitalization of aortic dissection in the National Inpatient Sample. J Vasc Surg. 64(3):606-615 e601. 